\title{
SKD: Keypoint Detection for Point Clouds using Saliency Estimation
}

\author{
Georgi Tinchev, Adrian Penate-Sanchez and Maurice Fallon
}

\begin{abstract}
We present SKD, a novel keypoint detector that uses saliency to determine the best candidates from a point cloud for tasks such as registration and reconstruction. The approach can be applied to any differentiable deep learning descriptor by using the gradients of that descriptor with respect to the 3D position of the input points as a measure of their saliency. The saliency is combined with the original descriptor and context information in a neural network, which is trained to learn robust keypoint candidates. The key intuition behind this approach is that keypoints are not extracted solely as a result of the geometry surrounding a point, but also take into account the descriptor's response. The approach was evaluated on two large LIDAR datasets - the Oxford RobotCar dataset and the KITTI dataset, where we obtain up to $50 \%$ improvement over the state-of-the-art in both matchability and repeatability. When performing sparse matching with the keypoints computed by our method we achieve a higher inlier ratio and faster convergence.
\end{abstract}

\section{INTRODUCTION}

A key task in robotics is the ability for a robot to localize itself in its surrounding environment. There are two prominent approaches - dense registration and sparse keypoint matching of points to a known map or object. Dense registration methods try to match every point to the map while sparse keypoint matching methods choose points with similar characteristics. Both methods have weaknesses: dense registration methods suffer from occlusions or modifications in the environment while keypoint matching requires a descriptive environment with repeatable points.

In this work, we are concerned about the challenges of sparse feature matching methods. Such methods are usually carried out in two stages: keypoint extraction, in which good points are selected according to some criteria, and keypoint description, in which the points are characterized. We present a novel method for keypoint extraction from point clouds that exploits the saliency of the point's descriptor to propose points with higher likelihood of being matched. This follows the recently proposed describe-then-detect methodology [1], where we focus mainly on the detect part, assuming an already trained differentiable point cloud descriptor.

Keypoint extraction methods have focused on analyzing the appearance of a sensor stream (mainly images or point clouds) so as to reliably generate keypoints at the same location when faced with different observation of the same place or object; this is known as repeatability. However, it presents

\footnotetext{
The authors are with the Oxford Robotics Institute, University of Oxford, United Kingdom. \{gtinchev, adrian, mfallon\}@robots.ox.ac.uk

This work was supported by EPSRC RAIN and ORCA Robotics Hubs (EP/R026084/1 and EP/R026173/1 respectively) and EU H2020 project Memory of Motion (MEMMO, project ID: 780684). M. Fallon is supported by a Royal Society University Research Fellowship.
}
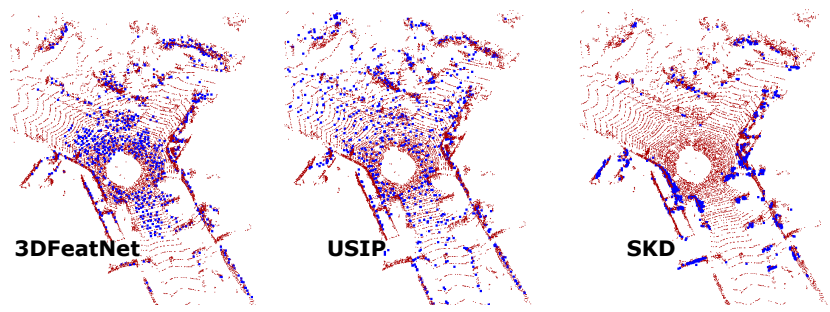

Fig. 1. Top-down view of a single point cloud (brown) and 1024 generated keypoints (blue). Our method (Salient Keypoint Detector, SKD) was not implicitly trained to ignore the ground, but rather it uses the feature signal of a descriptor network and the context information to determine informative areas in the environment - corners, edges and structure.

some issues depending on the particular object or place being analyzed. For example, when extracting visual keypoints and descriptors from a chessboard, keypoint extraction can be performed reliably and those points will be repeatable but the descriptors of those points will not be useful due to the ambiguity caused by the repeated pattern. The same can occur with point cloud data in which the windows of a building look alike. For this reason, recent research suggests that repeatability by itself is not sufficient for evaluating keypoint quality [2], [3]. A more reliable metric is matchability, as it measures how useful the combination of keypoint and feature is.

Our approach learns to produce points that can be reliably matched when given a pre-trained differentiable descriptor network. We leverage the idea presented in [3] for image data, in which state-of-the-art keypoint detectors can be built by only looking at the feature response. We use the gradient response of the descriptor network with respect to the input points as a measure of saliency, since it has been shown to produce promising results both in images and in point clouds [4], [5], [6]. Further, we combine the saliency with the original descriptor and context information in a neural architecture, which is trained to produce keypoints that generate a higher percentage of inlier matches, shown in Fig. 11. The main contributions of our work are as follows:

- The Salient Keypoint Detector (SKD): a novel method for extracting keypoints in point clouds based on the gradient response of a differentiable descriptor and the geometry of the scene.

- An environment agnostic approach: by using the gradient response rather than the actual point cloud, our approach is less vulnerable to biases in the training data. This makes our method robust when testing on different environments and allows the model to be used without retraining for different datasets.

- State-of-the-art performance: Compared to the stateof-the-art [9], [10], our approach generates two times 
more correct matches and achieves $40 \%$ more relative repeatability. We have evaluated our algorithm on two of the largest and most widely used LIDAR datasets - the Oxford RobotCar dataset [7] and the KITTI dataset [8]. In total these datasets contain point clouds collected from more than 300 kilometers of driving.

\section{RELATED WORK}

This paper focuses on reliably obtaining keypoints from point clouds. This is a topic which is very closely related to image keypoint extraction, so we will review the literature for both fields in this section. We will also outline the relevant research on point cloud-based deep learning models and saliency methods as these topics are central to the contributions of our approach.

\section{A. Image Keypoint Detectors}

Much of the recent work on keypoint and feature extraction from images employs deep learning architectures. In [11] a keypoint detector for depth images was presented. It used a Siamese approach by pairing two Faster-RCNN networks with a contrastive loss between them. Focusing on unsupervised keypoint learning, [12] presented an approach that generated reliable keypoints by learning from the temporal consistency of the network activations in short videos. A successful approach for obtaining good keypoints is to optimize how they respond to the content of the image and not just the geometry. TILDE [13] learned to make the points more reliable by understanding how changes in weather and lighting conditions modified the performance of a point. In LF-Net [14], the keypoint detector and the feature descriptor are jointly learned by leveraging structure from motion (SfM) sequences to generate large amounts of training data. By understanding that the job of a keypoint is to increase the probability of descriptor matching, it focused on matching performance and at the time outperformed all previous approaches. Recently, ELF [3] applied a simple approach by using the gradient response of deep learning features to produce keypoints.

R2D2 [2] not only focused on repeatability for a keypoint, but also on the reliability score based on the descriptor's response. They jointly learned the keypoint detector and the discriminativeness of the descriptor. This is similar to our proposition to look at matchability of a keypoint in addition to its repeatability. SuperPoint [15] jointly learned detection and description in a supervised task with multiple training stages on artificial images of basic structures. D2Net [1] used a CNN with shared weights for description and detection. In contrast to previous approaches it used a methodology of describe-then-detect. UnsuperPoint [16] improved the training methodology of SuperPoint by requiring only a single training round. They use a self-supervised detector and unsupervised descriptor to improve the repeatability of proposed keypoints. Their detector and descriptor also shared a common backbone. SIPs [17] introduced a metric to minimize the number of detected points with an iterative algorithm. Similar to us, they used the probability of a pixel being an inlier, but in 2D image data.

An alternative research direction was taken by GLAMpoints [18] to apply reinforcement learning rewards to SIFT matches in order to maximize correct correspondences. Similarly, DISK [19] leveraged cooperative reinforcement learning to match local features with a policy gradient. In contrast to the above works, we do not jointly train a keypoint detector and descriptor, but instead focus only on the former and assume a given keypoint descriptor. Therefore, our methodology can be viewed as similar to describe-thendetect, with a given differentiable describe method. Also, we do not explicitly define a reward for each correct keypoint, nor use any filtering techniques.

\section{B. Point Cloud Keypoint Detectors}

PointNet [20] and PointNet++ [21] introduced new ways to efficiently understand unstructured sets of points (point clouds). These works presented a novel layer for extracting descriptions from sets of points by learning a symmetric function and thus making the layer invariant to point order. PointCNN [22] managed to increase the performance of the basic neurons by approximating a convolution using an MLP and by performing aggregation of spatial data within each neuron. PointNetVLAD [23] presented a method to perform place recognition from point clouds. The point clouds were described using PointNet++ and NetVLAD trained with a metric learning loss to produce a feature vector. In contrast, we focus on detecting keypoints that aid descriptors such as [20], [21], [22]. Many recent papers leveraged PointNet layers in different applications [24], [6], [25]. In [6] the authors built a saliency map to understand the effect of each point on the final prediction. They tested the performance of their saliency score by performing point dropping operations to demonstrate performance better than a method based on the critical-subset theory [6]. We take inspiration from [6], but we do not perform point shifting or point dropping operations.

The most relevant methods to our work are 3DFeatNet [9] and USIP [10]. 3DFeatNet uses PointNet++ as a building block and learned a detector and a descriptor by using a two stage network. Unfortunately, the approach did not explicitly focus on good performance of the keypoint extraction network. USIP aimed to mitigate this explicitly by minimizing a variation of the Chamfer loss that focused on matching points reliably. In contrast, 3DFeatNet used a triplet loss, that focused in obtaining a descriptive feature space, but that cannot manage matching as it only focused on the descriptor. Compared to our work, 3DFeatNet [9] extracted attention for each point in the input, while we use the saliency scores obtained directly from the feature descriptor.

Recently, DH3D [26] introduced a hierarchical Siamese network for joint learning of local description, keypoint score, and global descriptor. It uses a coarse-to-fine relocalization technique to first find submaps in the scene and then local features for accurate pose estimation. Similarly to $3 \mathrm{DFeatNet}$, they used two-phase training to first train 

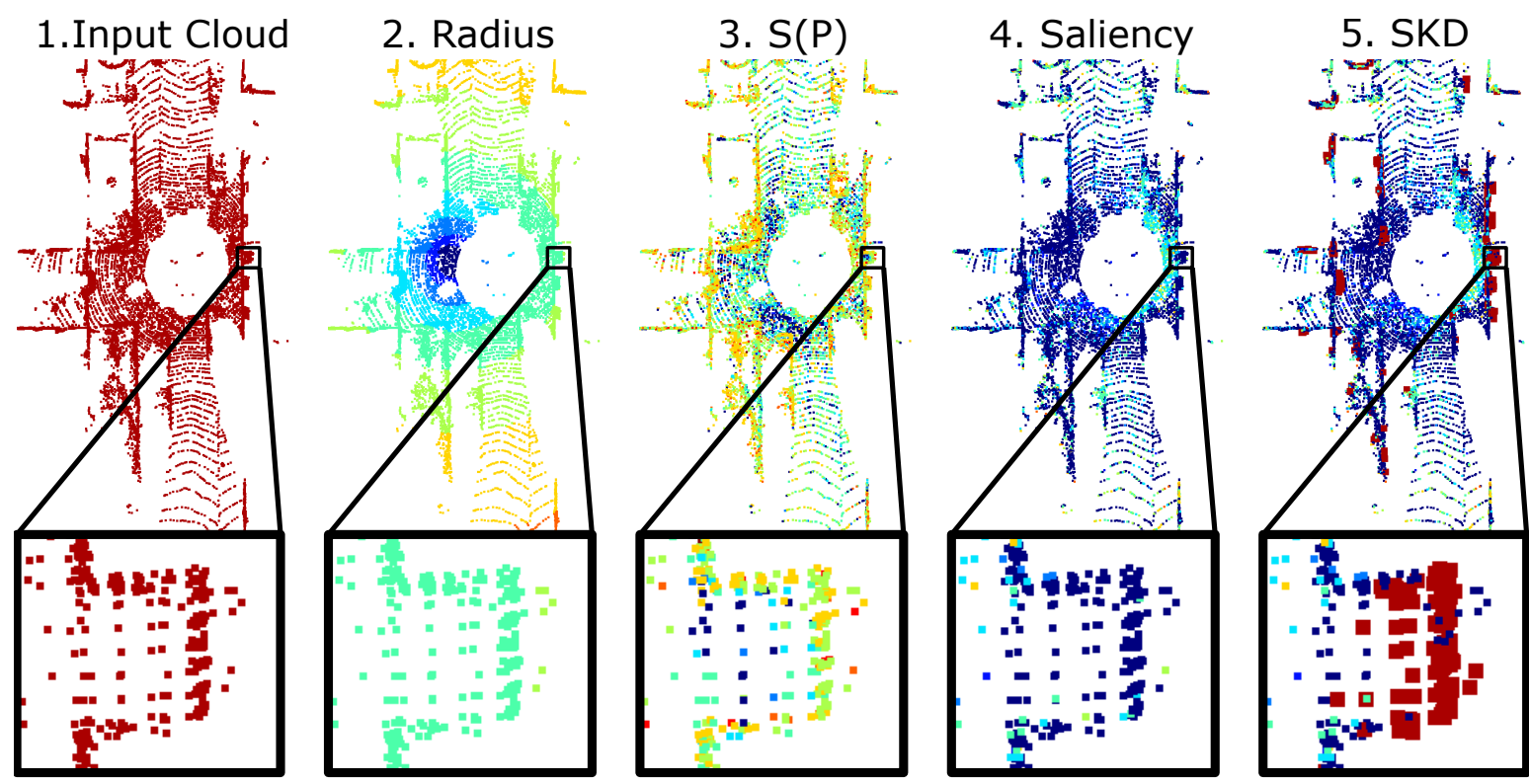

Fig. 2. 1. Input point cloud $P$. 2. Distances of each point $\left(r_{i}\right)$ from the point cloud median - blue indicates closer to the median point, red - further apart. 3. The initial saliency, $S(P)$, based on the activations and gradients w.r.t. input point cloud (1.) - blue corresponds to negative gradients, red highly positive. 4. The saliency score $s_{i}$ estimated from the radius (2.) and the gradients (3.), blue - low score, red - high score. 5. Selected keypoints (red, enlarged) on top of the saliency score. Our method picks keypoints based on the keypoint saliency score $s_{i}$, but also leverages context and the original descriptor to correctly pick out edges and corners negatively scored by $s_{i}$.

the descriptor and then the detector. D3Feat [27] adopt the joint descriptor and detector paradigm from D2-Net and extended it to point clouds. Their descriptor is trained using a contrastive loss, while a self-supervised loss is proposed for the detector, based on the descriptor's response. In contrast to these works we only train the keypoint detector while using a pre-existing descriptor.

\section{Saliency Estimation}

Saliency has been studied as a way to quantify and understand what is relevant within a neural network. The definition of saliency in this context has been studied by a number of works, such as [4], [28], [29] as a way to explain why machine learning models behave as they do. Even before the growth in popularity of neural networks, methodologies such as [30] were being designed to understand why classifiers made specific decisions, but became more prevalent with the adoption of deep learning models for most perception tasks. Approaches such as [4], [29] studied what a neural network finds relevant by looking at how the gradients of a given prediction behave. Their findings showed that gradients with higher magnitude in specific areas influence predictions.

The use of saliency has been demonstrated to be capable of generating state-of-the-art keypoint extractors in images. ELF [3] computed the gradient of the feature map given an image and then used the Kapur threshold [31] to select keypoints. In a similar fashion, Grad-CAM [28] used the gradient maps of a classification score to produce regions of interest for a given image for tasks such as classification, image captioning, and visual question answering. Inspired by these works, our approach applies saliency ideas to extract reliable keypoints on point cloud data.

\section{Methodology}

We present Salient Keypoint Detection (SKD) - a keypoint extraction method based on the saliency of a point cloud. Our approach follows describe-then-detect methodology to extract keypoints based on a pre-existing differentiable descriptor by the means of saliency. Both are combined with context information to using a neural network that learns to predict the likelihood of a point being a keypoint (Sec. IIIIB). Fig. 2 shows the main steps of our detector, which are further explained in the following sections.

\section{A. Point Cloud Saliency}

Given a point cloud $P=\left\{p_{i} \in \mathbb{R}^{3}\right\}^{N}$ with $N$ points and a pre-trained descriptor network $F$, we extract the gradients of the network at a specific layer $l$ with respect to the positional information of the input point cloud, defined as $\nabla F_{l}^{P} \in$ $\mathbb{R}^{\Omega_{l} \times N \times 3}$, where $\Omega_{l}$ is the feature map domain at layer $l$.

We define initial saliency $S(P)$, as the product of the feature activations of that layer $F_{l}^{P} \in \mathbb{R}^{\Omega_{l}}$ with the gradients, $\nabla F_{l}^{P}$. This is formally defined as:

$$
S(P)=F_{l}^{P} \cdot \nabla F_{l}^{P}
$$

where, $S(P) \in \mathbb{R}^{N \times 3}$. In this way the extracted initial saliency corresponds to specific points in the point cloud that have good activations and are valuable based on the gradient of that layer w.r.t. the input point cloud. From a geometric perspective this can be thought of as projecting the feature signal through to the input point cloud and determining the value of individual points (see example projection in Fig. 2).

We take the initial saliency computed from Eq. (1) and determine a secondary saliency score per point, defined as $s_{i}$ for each point. The score weighs the contribution of $S(P)$ 


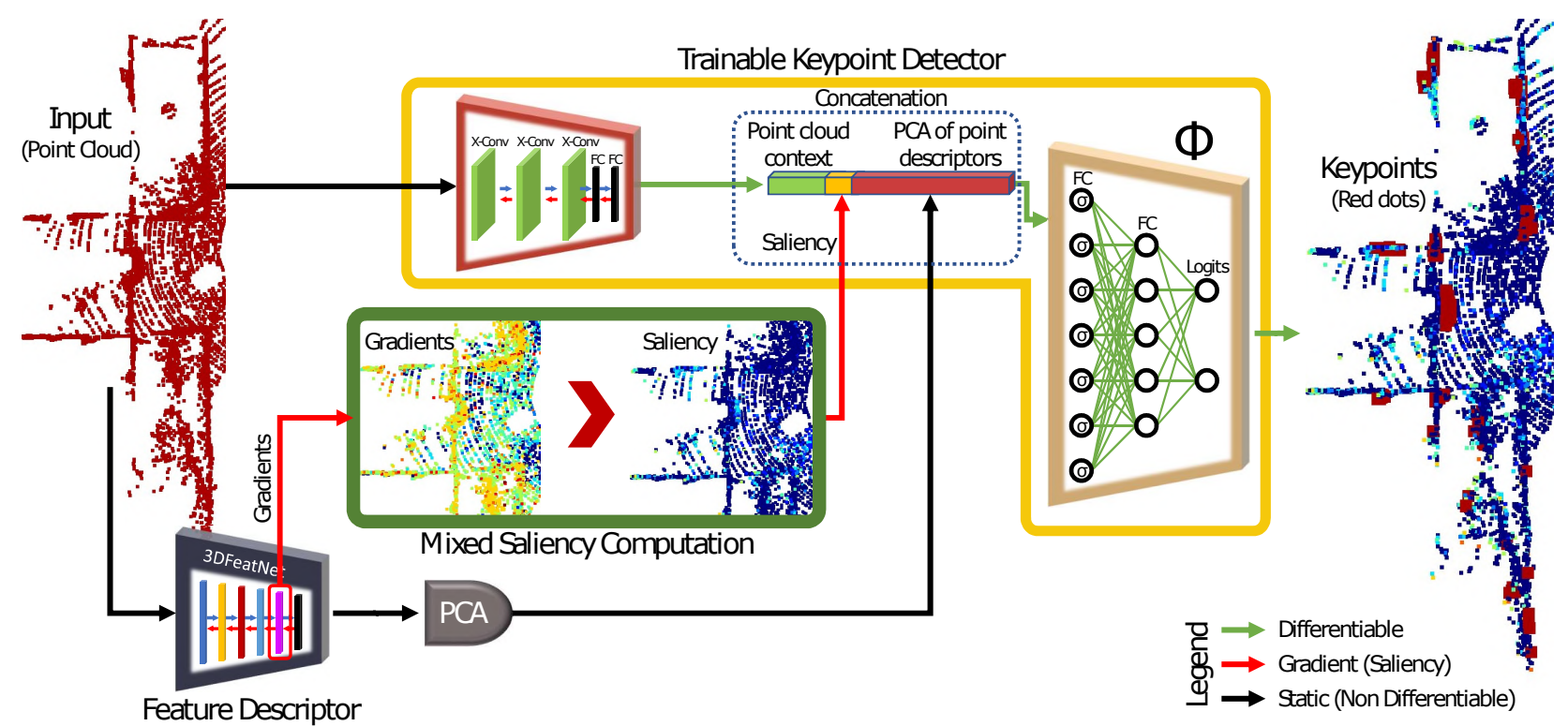

Fig. 3. Proposed network architecture. The input to our system is a raw point cloud and a feature descriptor network. Our method concatenates the saliency information from the gradients of the feature descriptor with the per-point context features and a PCA projection of the original feature descriptor. The concatenated vector is the input to two FC layers that generate per-point response at the logits layer.

by the distance from the median of the point cloud [6]. The score is formally defined as:

$$
s_{i}=-\sum_{j=1}^{3}\left[S_{j}(P) \cdot\left(x_{i j}-\operatorname{median}\left(x_{i j}\right)\right)\right] r_{i}
$$

where $j \in\{1,2,3\}$ defines each of the Cartesian coordinates $x, y, z$ of point $p_{i} \in P$ and $r_{i}=$ $\sqrt{\sum_{j=1}^{3}\left(x_{i j}-\operatorname{median}\left(x_{i j}\right)\right)^{2}}$ is the distance of point $i$ to the median of the point cloud. Therefore, further away points will have higher score, and points closer to the center will have lower score (see Fig. 2). This design decision is motivated by the high density of points near the origin of the sensor (see distribution of points of 3DFeatNet vs SKD in Fig. 7). Also, note that well distributed keypoints in two point clouds will likely result in a more robust solution.

Finally, in accordance with standard deep learning practices, the input saliences are normalized to have zero mean and unit variance within a single point cloud. The final saliency ensures a good spatial distribution of the selected points while also selecting points with good activations based on the descriptor network. Fig. 2 illustrates the saliences of an example input point cloud at each stage of computation.

\section{B. Network Architecture}

In order to determine the keypoints, we designed a neural network to combine the saliences with other sources of information. Our architecture is depicted in Fig. 3 and consists of three modules that generate a combined feature vector, which is the input for a final fully-connected network used to compute the likelihood of each point being a keypoint.

- The first component, mixed saliency computation, is the saliency method described in the previous section.
- The second component is a PCA dimensionality reduction of the original per-point features. This improved the final results by producing a smoother feature space.

- The third part is called the point cloud context features. These are four X-Conv layers [22] and two fully connected (FC) layers that have been pretrained on a feature extraction task to create stable initial estimates. These layers learn to provide a description of the context around any given point. We use a two dimensional size for the context latent space.

The output of the three components are concatenated and fed to two additional fully connected layers, denoted by $\Phi$, to produce the final keypoint prediction. The network learns to infer a score for each point - determining the likelihood of it being a robust and repeatable keypoint for the original descriptor. Note that our model is descriptor-agnostic and thus can be applied to any descriptor network in order to improve the performance.

1) Training: During training, the input to our model consists of a pair of point clouds and the ground truth transformation between them, $\left(P_{k}, P_{l}, T\right)$. Both $P_{k}$ and $P_{l}$ are $N \times 3$ dimensional vectors, where $N$ denotes the cardinality of the point cloud set. In addition, we assume to have a pre-trained model for the point descriptors. To train we estimate the saliency $s_{i}$ and features $f_{i}$ for each point in both point clouds. Due to the large dimensionality of the feature space $f_{i}$, we performed PCA and transformed the features that explained $\approx 90 \%$ of the data. In addition, given the ground truth transformation between the two point clouds, we determine the bidirectional correspondence for each point, conditioned on the descriptor. These correspondences are used in our loss to select matching pairs of points. These correspondences need not be injective nor surjective: if for a point $i_{1}$ the closest neighbour under the ground truth transformation (up to a $1 \mathrm{~m}$ threshold) is $j_{1}$, it does not mean the reverse applies 


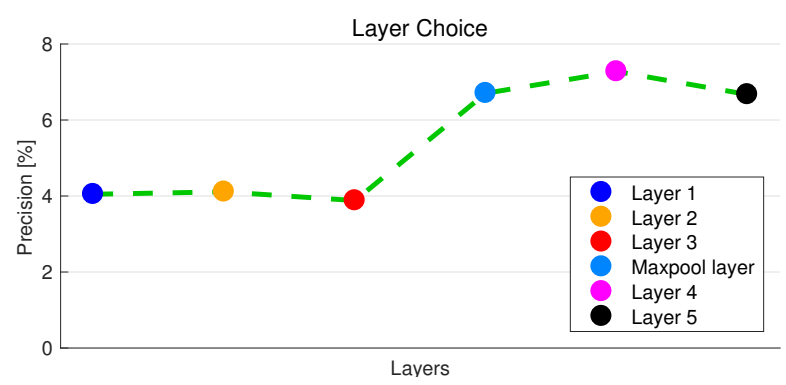

Fig. 4. Performance of each layer of 3DFeatNet expressed as percentage of correct matches if the layer was selected for gradient computation. The layer identified corresponds to the counter of layers in [9] starting from the left. In our evaluation we used the layer that maximized the performance (Layer 4) for both ELF [3] and our method.

nor that $i_{1}$ has a unique neighbour from $P_{l}$.

For each point in both point clouds we extract contextaware features, $f_{c}$, and concatenate them to the saliencies $s_{i}$ and features $f_{i}$. The term context-aware is used as we expect the layers that lead to this descriptor to contain information about the local geometry around each point. We pretrain these layers on a feature learning task to obtain stable initial features in the training. We chose a small feature space of only two dimensions in order to force the network to learn rough estimates of the shape of objects that can generalize better when moving to a different dataset. Afterwards, the full architecture is trained end-to-end with the saliency and feature concatenation. We note that the contribution of the context-aware features is smaller compared to $s_{i}$ and $f_{i}$.

The concatenated saliences, PCA features, and contextaware features are fed to two fully connected layers to estimate the likelihood of each of the points being a keypoint. To this end we use a softmax cross entropy loss between the stacked $P_{k}, P_{l}$ clouds and the determined correspondences, given the ground truth transformation. Due to the smaller number of correct keypoint correspondences between the two clouds, we balance the loss function terms given the keypoint to non-keypoint ratio determined by the ground truth correspondences.

2) Inference: During the forward pass of the network we estimate a likelihood for each point being a correct keypoint. We can then either extract the top $K$ keypoints based on this likelihood or select all the keypoints above certain threshold. The extracted keypoints produced by the described approach are better suited to the descriptor as the learning iterations optimize its performance. Note, that neither Non-Maximum Suppression, nor any other threshold is applied to filter the final set of keypoints.

\section{RESUlTS}

Next, we discuss the datasets, baselines, and metrics we used to evaluate our approach and then present our findings.

\section{A. Datasets}

In our study we used two datasets - the Oxford RobotCar [7] and the KITTI odometry dataset [8]. In order to provide a fair comparison, our experiments use the preprocessed test data provided by related previous works, [9] and [10]. We also used their evaluation scripts to make the comparison as clear as possible. We trained our model using the same sequences from the RobotCar dataset as [9], and tested our approach using the same test set of 3,426 point cloud pairs which the authors provided. Furthermore, we neither trained our method nor these baselines on the KITTI dataset, in order to test the generalization ability of the approaches.

The evaluation part of the KITTI dataset, used by both [9], [10], provides only 2,369 point clouds out of the total dataset. So as to increase the size of the KITTI evaluation dataset, we extended it using the 11 training sequences. This is possible only because the RobotCar dataset is used for training all models. The extended dataset is processed in a dense manner: for each point cloud we align the next consecutive 10 point clouds to it using the ground truth sensor poses. By doing this we expanded the number of testing point cloud pairs from 2,831 to 207,917, which allows us to more fully study our proposed approach. Note that none of the KITTI data was used for training our method, any of the baselines, or the descriptor.

\section{B. Metrics}

We focused our analyses on the keypoint extraction methods and compared them using three metrics. To ensure a fair comparison to the baseline keypoint extraction methods (see Sec. IV-C), we used the same 3DFeatNet [9] descriptor for all the methods.

The first metric used the matching score proposed by [9] that determined correspondences within $1 \mathrm{~m}$ maximum distance. We detected keypoints separately for two point clouds. Given the ground truth sensor poses, we projected the keypoints from the first point cloud into the second one. Keypoints that did not have a nearest neighbour bidirectional correspondence in the second point cloud were ignored from the final result (i.e. no overlap). For the rest of the keypoints, the descriptors were compared and matched to establish a correspondence. The precision was measured as the number of correct correspondences against the total number of possible matches. This metric estimated the percentage of correct correspondences based on the distance between them. Correct correspondences were thresholded to $1 \mathrm{~m}$ distance, as it served as a desirable upper limit when performing registration between two point clouds.

For the second metric we chose to compare the normalized relative repeatability, as proposed in [10]. The metric compared the keypoints detected in one point cloud to the keypoints detected in its corresponding point cloud. If the keypoints overlapped, below a certain distance, they were considered a match. While this saturated with a high number of keypoints [3], we chose to use this metric to have a fair comparison against USIP [10]. We note that the ability to match keypoints on their own is not sufficient, hence we considered the matching score to be the more important metric.

Finally, we evaluated the geometric registration performance of our approach on both the KITTI and the Oxford RobotCar datasets using RANSAC [32]. As in [9], we consider an alignment to be successful if it differs by less 

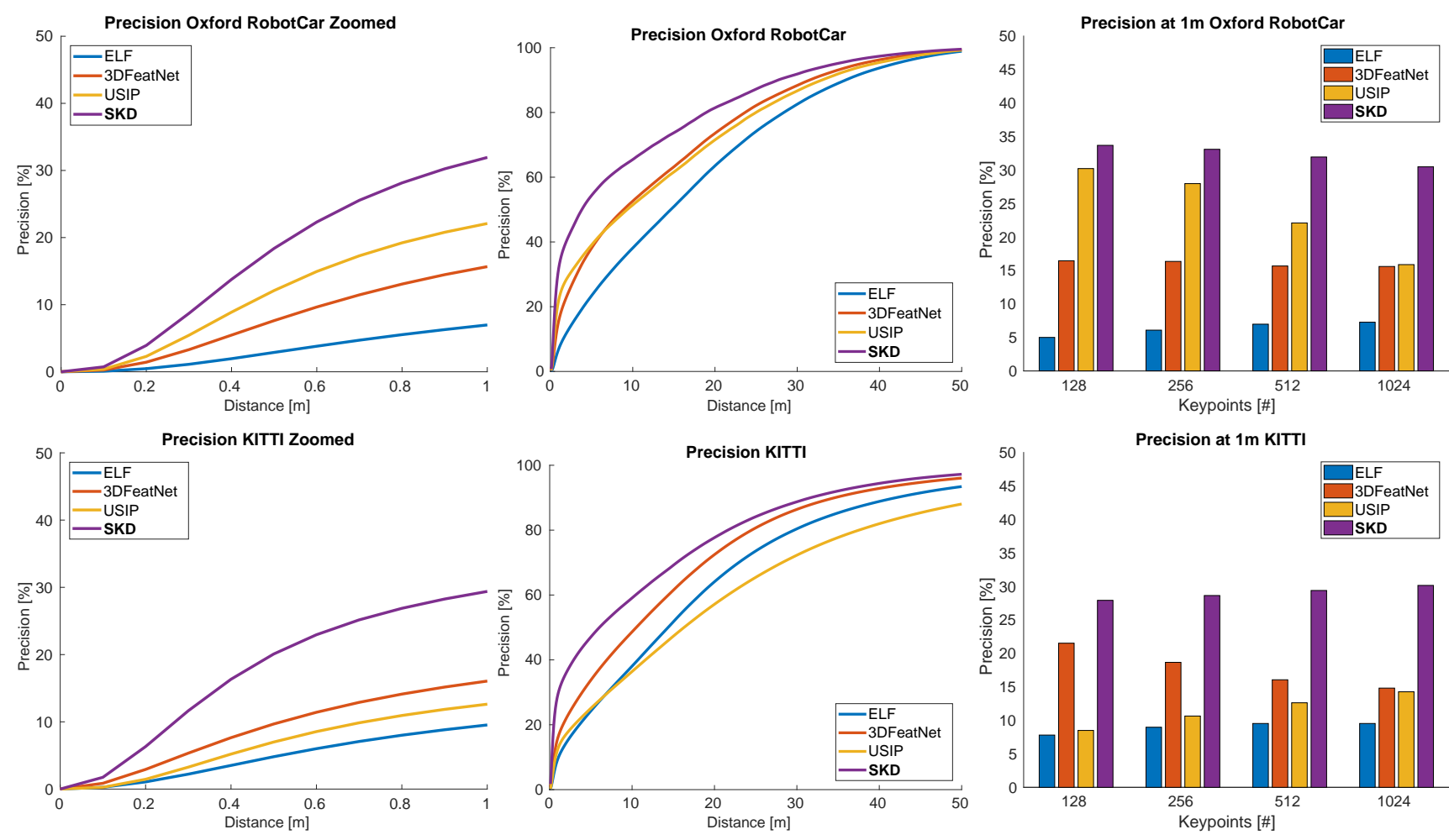

Fig. 5. Matching score evaluated on the Oxford RobotCar dataset (top row) and the KITTI dataset (bottom row). The first two columns are the percentage of matched keypoints using the 3DFeatNet [9] descriptor when varying the distance between correspondences. The first column shows a zoomed-in version of the second column within $1 \mathrm{~m}$ of distance, considered relevant for geometric registration. The third column shows the performance of different approaches while varying the number of keypoints.

than $2 \mathrm{~m}$ and $5^{\circ}$ from the ground truth sensor pose. For the successful registrations we present relative translation error (RTE), relative rotation error (RRE), success rate as a percentage of the successful registrations, average number of iterations it took RANSAC to find a suitable candidate within 99\% confidence (capped at 10,000 iterations), and the inlier ratio of how many points were considered when obtaining a correct registration.

\section{Baseline Methods}

Our approach is general and can be applied to any point cloud descriptor network. For simplicity we chose to use the descriptor [9] as it is open source and easy to use, as well as being state-of-the-art point cloud descriptor. We select the best performing layer from the descriptor to generate the gradients on which we compute the saliency values, as illustrated in Fig. 4. We compared against the learned keypoint detector methods of 3DFeatNet [9], DH3D [26], USIP [10], and a 3D interpretation of the ELF [3] detector. All the learning methods were trained on the Oxford RobotCar dataset and tested on both RobotCar and KITTI data. We have used the models provided online, and trained our own network. For USIP, we took the models provided by the authors which they trained on the Oxford RobotCar dataset. As ELF does not need training, we took the best performing layer, according to Fig. 4 . We adapted their approach to work on point clouds by performing NonMaximum Suppression in 3D and choosing keypoints based on the Kapur Threshold [31].

\section{Matching Score Experiments}

We evaluated our approach using the matching score metric on both Oxford RobotCar and KITTI datasets. Fig. 5 illustrates the performance of SKD compared to other stateof-the-art methods. The first row shows the percentage of matched keypoints on the Oxford RobotCar dataset using the 3DFeatNet descriptor for all methods. The first column is a zoomed-in version of the percentage of points detected within $1 \mathrm{~m}$ distance that are considered relevant for matching. In the second column we show the full results as presented in [9]. The last column shows the precision at $1 \mathrm{~m}$ distance at different numbers of keypoints - 128, 256, 512, 1024. As all the approaches are learning-based, we took the top $K$ keypoints; this ensures that the number of keypoints are identical. The second row presents the results for the full KITTI dataset, with all the methods trained on the Oxford RobotCar dataset. We observe that our approach generalizes well without a loss in performance, and outperforms the second best approach by a significant margin. Similarly to 3DFeatNet [9], our approach's performance does not decline with the increase of detected keypoints.

\section{E. Repeatability Experiments}

Next, we evaluate the repeatability of keypoints across multiple observations of the same scene. Fig. 6 shows the repeatability of SKD measured on the Oxford RobotCar dataset (left) and the KITTI dataset (right). For the Oxford 

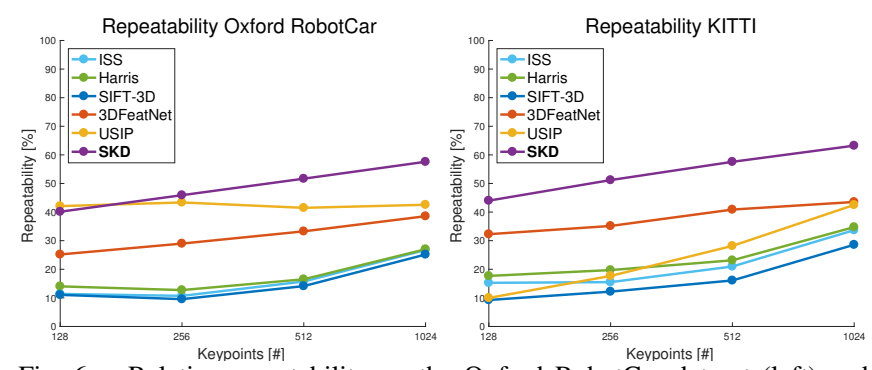

Fig. 6. Relative repeatability on the Oxford RobotCar dataset (left) and KITTI (right) with different number of extracted keypoints.

RobotCar, our approach performs comparably to the second best method when extracting 128 and 256 keypoints, but it selects points that are $40 \%$ more repeatable when extracting 1024 interesting points. Furthermore, Fig. 1, 7, 8, and the supplementary video material present qualitative examples of the detection of keypoints of the top three approaches. The proposed approach has learned to select more descriptive areas of the environment. For example, our method does not select ground points, and even though we forced the method to generate large amounts of points, it still chooses points based on the high activations of the network - around building edges and corners, that in turn are easier to match.

Fig. 6 (right) illustrates the repeatability of our approach on the KITTI dataset. On this dataset, 3DFeatNet performs slightly better than on the Oxford RobotCar dataset. Interestingly, as we use the feature signal of 3DFeatNet, our keypoint detector also performs better. In addition, Fig. 7 presents qualitative evaluation of our approach on the KITTI dataset with an increased number of keypoints, where SKD consistently selects the same areas of the environment.

\section{F. Geometric Verification Experiments}

Although our work is focused on keypoint detection, we show results for one possible application of our detector - geometric registration. Tab. II presents the results of geometric registration on KITTI and Oxford RobotCar. We used the baselines' official code and models, but note that we used 73 times more evaluation data for KITTI.

SKD performs similarly to the state-of-the-art in terms of relative rotation and translation error, within the standard deviation of the best-performing method $\left(2 \mathrm{~mm}\right.$ and $0.04^{\circ}$ difference with best baseline). Our method is, however, approximately nine times faster on the KITTI dataset and two times faster on the Oxford RobotCar dataset compared to the second-best approach with a higher percentage of inliers. We note that we did not use non-maximum suppression (NMS), which we suspect is the reason for the slightly higher errors and lower success rate. Applying NMS first and then extracting the top $\mathrm{K}$ keypoints would reduce the number of grouped keypoints, and as such the robust estimator could find better distributed keypoints, which will likely result in better point cloud registration accuracy. As shown in our repeatability and matchability experiments, our method is good at picking out keypoints at similar locations. However, for applications such as global registration highly repeatable and matchable points are not the only necessary requirement.

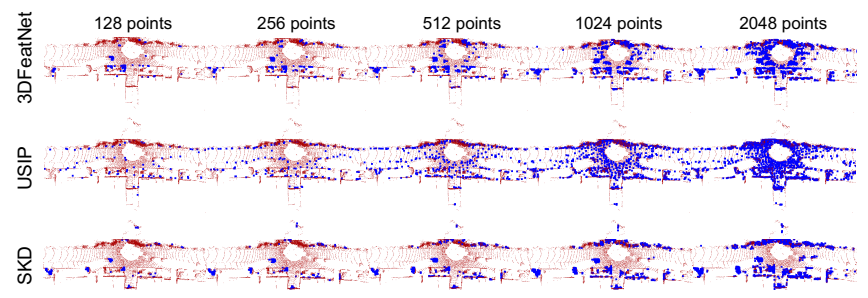

Fig. 7. Qualitative results on the KITTI dataset. For all methods the number of generated keypoints is increased from left to right. This shows what relative importance each method gives to certain areas of the point cloud.

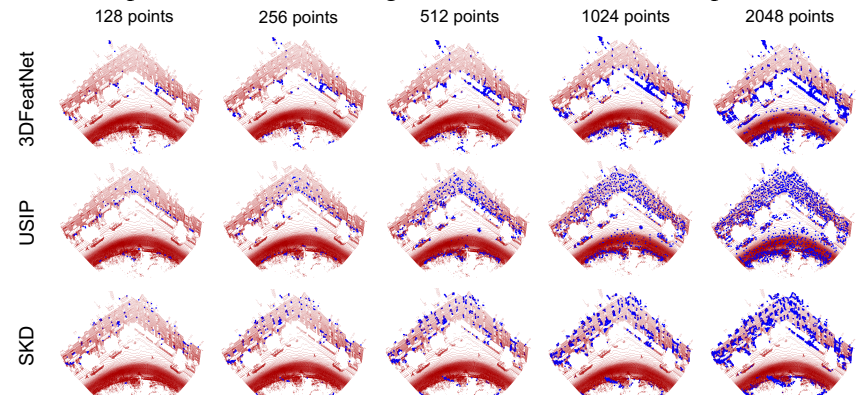

Fig. 8. Qualitative results on the Oxford RobotCar dataset. The keypoint proposals from SKD are evenly spread in geometrically interesting locations.

In contrast, for object pose estimation highly repeatable points are arguably more important than the distribution of points. Furthermore, not using NMS makes our detector more robust to changes in the scene.

\section{G. Ablation studies}

Next, we studied the contribution of each component of our method by measuring the precision at $1 \mathrm{~m}$ distance on the Oxford RobotCar dataset, shown in Fig. 9 Note that the saliency can be extracted without any training and thus we present ablation studies with both options.

First, we inspected the contribution of the saliency in the concatenated vector. In our experiments the saliency information alone is responsible for approximately half of the concatenated vector - dark blue compared to yellow in Fig. 9 In addition, training the neural network, $\Phi$, with just saliency information contributes to approximately a third of the end result - red compared to light blue, thus justifying the name of our method. Second, the contribution of the network, $\Phi$, when training with the full feature vector, is substantial as is to be expected - purple compared to dark blue and yellow on Fig. 9 By analyzing our ablation study in Fig. 9 we can see that the increase in performance from the context features is only marginal. We would like to note that the performance gains, and thus the contributions of this paper, come from using saliency together with the value of the feature to determine which points to extract as keypoints. Finally the use of PCA was introduced to smooth the feature space and allow for better generalization, and improved performance, as can be seen in in Fig. 9 (green vs light blue).

\section{Conclusions}

In this paper we presented a novel method for keypoint extraction that uses saliency information to extract informative regions in a point cloud. The method concatenates 


\begin{tabular}{|c|c|c|c|c|c|c|}
\hline & Detector + Descriptor Method & RTE (m) & $\operatorname{RRE}\left({ }^{\circ}\right)$ & Success Rate & Avg \# iter & Inlier ratio \\
\hline \multirow{5}{*}{$\frac{\overrightarrow{0}}{0}$} & ELF [3] + 3DFeatNet [9] $*$ & $0.42 \pm 0.31$ & $1.66 \pm 1.09$ & $86.49 \%$ & 9788 & $5.3 \%$ \\
\hline & 3DFeatNet [9] + 3DFeatNet [9] * & $0.30 \pm 0.25$ & $1.07 \pm 0.85$ & $97.64 \%$ & 3083 & $12.9 \%$ \\
\hline & USIP [10] + 3DFeatNet [9] * & $0.29 \pm 0.26$ & $0.96 \pm 0.77$ & $\mathbf{9 8 . 7 4 \%}$ & 823 & $21.0 \%$ \\
\hline & DH3D [26] + 3DFeatNet [9] & 0.28 & 1.04 & $98.2 \%$ & 2908 & - \\
\hline & SKD + 3DFeatNet [9] & $0.31 \pm 0.27$ & $1.11 \pm 0.89$ & $97.64 \%$ & 393 & $\mathbf{3 2 . 7} \%$ \\
\hline \multirow{3}{*}{$\vec{E}$} & 3DFeatNet [9] + 3DFeatNet [9] * & $0.142 \pm 0.120$ & $0.533 \pm 0.410$ & $\mathbf{9 7 . 8 0 \%}$ & 3917 & $12.7 \%$ \\
\hline & USIP [10] + 3DFeatNet [9] $*$ & $0.203 \pm 0.193$ & $0.637 \pm 0.517$ & $97.12 \%$ & 5324 & $11.0 \%$ \\
\hline & SKD + 3DFeatNet [9] & $0.140 \pm 0.134$ & $0.579 \pm 0.480$ & $96.52 \%$ & 594 & $\mathbf{3 2 . 2} \%$ \\
\hline
\end{tabular}

TABLE I: Geometric registration on the Oxford RobotCar dataset (top) and the KITTI dataset (bottom), evaluated by RANSAC. * indicates our evaluation on the dataset using each method's official implementation and models. Note: the used KITTI dataset is 73 times larger than the one by [9], [10].

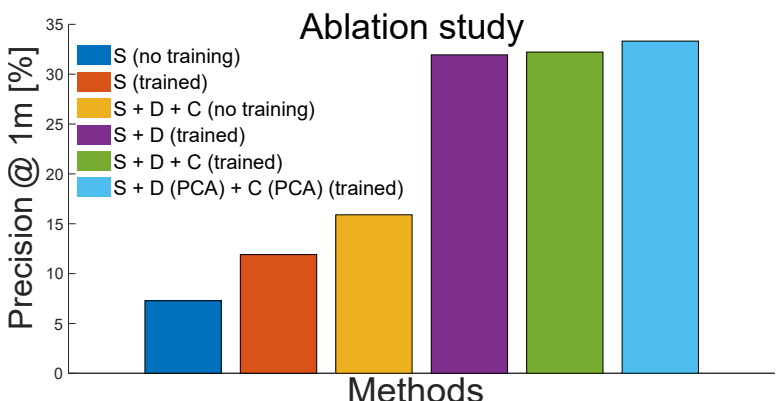

Fig. 9. $\mathrm{S}=$ Saliency, $\mathrm{D}=$ Descriptor, $\mathrm{C}=$ Context. Ablation studies of our method evaluated on the Oxford RobotCar dataset. We evaluate the contribution of the saliency information, and the importance of training with the original descriptor. The results displayed in the figure show the clear contribution the neural network is making to the keypoint generation.

saliency computed from the gradient of a pre-trained descriptor network evaluated at the input cloud, context-aware features, and the descriptor features and learns to predict which 3D points have a higher chance of being matched correctly. The proposed approach is descriptor-agnostic and outperforms the state-of-the-art by up to $50 \%$ in matchability and repeatability compared to the second-best method. When performing geometric registration, the algorithm achieves a higher number of inliers while also finding a correct pose estimate significantly faster than the current state-of-the-art.

\section{ACKNOWLEDGMENTS}

We would like to thank Tom Dobra for maintaining the resources used for this project. We are also grateful for the detailed corrections by Matias Mattamala.

\section{REFERENCES}

[1] M. Dusmanu, I. Rocco, T. Pajdla, M. Pollefeys, J. Sivic, A. Torii, and T. Sattler, "D2-Net: a trainable CNN for joint description and detection of local features," in CVPR, 2019, pp. 8092-8101.

[2] J. Revaud, P. Weinzaepfel, C. R. de Souza, and M. Humenberger, "R2D2: repeatable and reliable detector and descriptor," in NIPS, 2019.

[3] A. Benbihi, M. Geist, and C. Pradalier, "ELF: embedded localisation of features in pre-trained CNN," in ICCV, 2019.

[4] K. Simonyan, A. Vedaldi, and A. Zisserman, "Deep inside convolutional networks: Visualising image classification models and saliency maps," in ICLRW, 2014

[5] J. Springenberg, A. Dosovitskiy, T. Brox, and M. Riedmiller, "Striving for simplicity: The all convolutional net," in ICLRW, 2015.

[6] T. Zheng, C. Chen, J. Yuan, and K. Ren, "Pointcloud saliency maps," in ICCV, 2019.

[7] W. Maddern, G. Pascoe, C. Linegar, and P. Newman, "1 Year, 1000km: The Oxford RobotCar Dataset," IJRR, vol. 36, no. 1, pp. 3-15, 2017.

[8] A. Geiger, P. Lenz, C. Stiller, and R. Urtasun, "Vision meets robotics: The KITTI dataset," IJRR, vol. 32, no. 11, pp. 1231 - 1237, 2013.

[9] Z. J. Yew and G. H. Lee, "3dfeat-net: Weakly supervised local 3d features for point cloud registration," in ECCV, 2018.
[10] J. Li and G. H. Lee, "Usip: Unsupervised stable interest point detection from 3d point clouds," in ICCV, 2019.

[11] G. Georgakis, S. Karanam, Z. Wu, J. Ernst, and J. Košecká, "End-toend learning of keypoint detector and descriptor for pose invariant $3 \mathrm{~d}$ matching," in CVPR, 2018.

[12] T. Kulkarni, A. Gupta, C. Ionescu, S. Borgeaud, M. Reynolds, A. Zisserman, and V. Mnih, "Unsupervised learning of object keypoints for perception and control," in NeurIPS, 2019.

[13] Y. Verdie, K. M. Yi, P. Fua, and V. Lepetit, "TILDE: A temporally invariant learned detector," in CVPR, 2014.

[14] Y. Ono, E. Trulls, P. Fua, and K. M. Yi, "Lf-net: Learning local features from images," in NeurIPS, 2018.

[15] D. DeTone, T. Malisiewicz, and A. Rabinovich, "Superpoint: Selfsupervised interest point detection and description," in CVPR, 2018.

[16] P. H. Christiansen, M. F. Kragh, Y. Brodskiy, and H. Karstoft, "UnsuperPoint: end-to-end unsupervised interest point detector and descriptor," arXiv preprint arXiv:1907.04011, 2019.

[17] T. Cieslewski, K. G. Derpanis, and D. Scaramuzza, "SIPs: succinct interest points from unsupervised inlierness probability learning," in 3DV. IEEE, 2019, pp. 604-613.

[18] P. Truong, S. Apostolopoulos, A. Mosinska, S. Stucky, C. Ciller, and S. D. Zanet, "GLAMpoints: greedily learned accurate match points," in ICCV, 2019, pp. 10732-10741.

[19] M. Tyszkiewicz, P. Fua, and E. Trulls, "DISK: learning local features with policy gradient," NeurIPS, vol. 33, 2020.

[20] C. R. Qi, H. Su, K. Mo, and L. J. Guibas, "PointNet: Deep Learning on Point Sets for 3D Classification and Segmentation," in CVPR, 2017.

[21] C. R. Qi, L. Yi, H. Su, and L. J. Guibas, "PointNet++: Deep Hierarchical Feature Learning on Point Sets in a Metric Space," in NeurIPS, 2017.

[22] Y. Li, R. Bu, M. Sun, W. Wu, X. Di, and B. Chen, "Pointenn: Convolution on x-transformed points," in NeurIPS, 2018.

[23] M. A. Uy and G. H. Lee, "PointNetVLAD: Deep Point Cloud Based Retrieval for Large-Scale Place Recognition," in CVPR, 2018.

[24] C. Xiang, C. R. Qi, and B. Li, "Generating 3D adversarial point clouds," in CVPR, June 2019.

[25] D. Xu, D. Anguelov, and A. Jain, "PointFusion: Deep sensor fusion for 3D bounding box estimation," in CVPR, 2018.

[26] J. Du, R. Wang, and D. Cremers, "DH3D: deep hierarchical 3D descriptors for robust large-scale $6 \mathrm{DoF}$ relocalization," in ECCV. Springer, 2020, pp. 744-762.

[27] X. Bai, Z. Luo, L. Zhou, H. Fu, L. Quan, and C.-L. Tai, "D3Feat: joint learning of dense detection and description of 3D local features," in CVPR, 2020, pp. 6359-6367.

[28] R. R. Selvaraju, M. Cogswell, A. Das, R. Vedantam, D. Parikh, and D. Batra, "Grad-cam: Visual explanations from deep networks via gradient-based localization," in ICCV, Oct 2017.

[29] J. Adebayo, J. Gilmer, M. Muelly, I. Goodfellow, M. Hardt, and B. Kim, "Sanity checks for saliency maps," in NeurIPS, 2018.

[30] D. Baehrens, T. Schroeter, S. Harmeling, M. Kawanabe, K. Hansen, and K.-R. Müller, "How to explain individual classification decisions," J. Mach. Learn. Res., vol. 11, pp. 1803-1831, Aug. 2010.

[31] J. Kapur, P. Sahoo, and A. Wong, "A new method for gray-level picture thresholding using the entropy of the histogram," Computer Vision, Graphics, and Image Processing, vol. 29, no. 3, pp. 273 - 285, 1985

[32] M. A. Fischler and R. C. Bolles, "Random sample consensus: A paradigm for model fitting with applications to image analysis and automated cartography," CACM, vol. 24, no. 6, pp. 381-395, 1981. 